\title{
Identidad Nacional en la generación posmillenial peruana
}

\author{
National Identity in the Peruvian post-millennial generation \\ Identidade nacional na geração peruana pós-milenar
}

\section{ARTÍCULO GENERAL}

\author{
Álvaro Adrián Rodríguez Barriga \\ alvaro.rodriguez@ucsm.edu.pe \\ https://orcid.org/0000-0003-4783-016X \\ Universidad Católica de Santa María, Perú
}

Recibido 15 de Febrero 2021 | Arbitrado y aceptado 03 de Marzo 2021 | Publicado el 01 de Mayo 2021

\begin{abstract}
RESUMEN
Considerando los aspectos descriptores de la identidad nacional, en el presenta artículo se busca terminar la percepción de identidad nacional de estudiantes de la Universidad Católica Santa María de la ciudad de Arequipa que tengan como característica pertenecer a la generación posmillennials con edades entre los 25 y 14 años. Para el logro del objetico, se adoptó una metodología cuantitativa de diseño no experimental y transversal de tipo descriptivo, aplicando un instrumento tipo cuestionario de escala Likert diseñado con cuatro dimensiones: simbólica valorativa, económica, política y cultural, aplicado a 376 estudiantes matriculados en el año 2020; producto de la investigación realizada por Rodriguez (2020). Obteniendo una característica generacional de identidad nacional marcada por la neutralidad y la preferencia por productos, marcas y cultura extranjera.

Palabras claves: generación, identidad nacional, peruana, posmillennials.
\end{abstract}

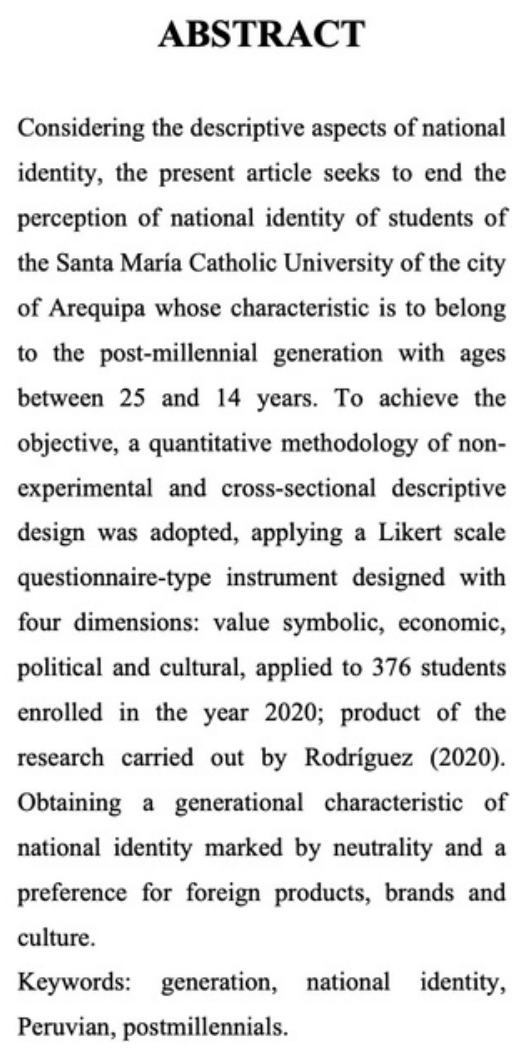

\section{ABSTRACT}

Considering the descriptive aspects of national identity, the present article seeks to end the perception of national identity of students of the Santa Maria Catholic University of the city of Arequipa whose characteristic is to belong to the post-millennial generation with ages between 25 and 14 years. To achieve the objective, a quantitative methodology of nonexperimental and cross-sectional descriptive design was adopted, applying a Likert scale questionnaire-type instrument designed with four dimensions: value symbolic, economic, political and cultural, applied to 376 students enrolled in the year 2020; product of the research carried out by Rodríguez (2020). Obtaining a generational characteristic of national identity marked by neutrality and a preference for foreign products, brands and culture.

Keywords: generation, national identity, Peruvian, postmillennials.

\section{RESUMO}

Considerando os aspectos descritivos da identidade nacional, o presente artigo busca encerrar a percepção da identidade nacional dos estudantes da Universidade Católica Santa María da cidade de Arequipa cuja característica é pertencer à geração pós-milenar com idades entre 25 e 14 anos. Para atingir o objetivo, adotou-se uma metodologia quantitativa de desenho descritivo não experimental e transversal, aplicando um instrumento tipo questionário de escala Likert elaborado com quatro dimensões: valor simbólico, econômico, político e cultural, aplicado a 376 alunos matriculados no ano 2020; produto da pesquisa realizada por Rodríguez (2020). Obtenção de uma característica geracional de identidade nacional marcada pela neutralidade e pela preferência por produtos, marcas e cultura estrangeiras.

Palavras-chave: geração, identidade nacional, peruano, pós-milenistas. 


\section{Introducción}

La sensación de pertenencia a un grupo familiar, social, educativo y político es esencial en la acción social del ser humano; desde los primeros años de infancia hasta la adultez; y desde todas las facetas de la vida. El ser social tiene repercusiones personales y colectivas; siendo estas de índole positiva y negativa, o ambivalentes. Desde que se nace y comienza a interactuar, la formación de la personalidad, de los valores familiares, de los valores naciones; constituyen los componentes del ser social (Cueto, Espinosa y Robles, 2017), y con ello la identificación o percepción de pertinencia.

Es posible, concebir una persona integrante de una familia "convencional" pero no considerarse integrante de un grupo social, por ejemplo: amigos, compañeros de trabajo; no es difícil imaginar las situaciones a la cuales hará frente; el compartir objetivos, metas, gustos y/o hobbies hacen que la persona sea socialmente integrada; pero si la transcendencia de su integración no alcanzan preponderar fines comunes desde algo aún más grande que una organización laboral; sino más bien de un integración con la nación a la cual pertenece tanto por nacimiento como por su actividad económica.

En este punto, la integración al gentilicio y a la nacionalidad; permiten a ese ser social formar un conjunto de valores y costumbres que son únicas e identitarias de la región geográfica que le ofrece influencia; a modo análogo, es ese integrante no se identifica por los objetivos, valores y metas colectivas de cierto territorio, es posible, ¿qué ese ser social tenga identidad cultural, social, territorial y política por su nación? La adaptación a un entorno dónde las situaciones sociales, económicas, políticas y culturales propician el descontento y la falta de integración de un sujeto por la nación donde habita, hacen atribuirles a tales efectos, y causas, un carácter sistémico y complejo (Pastor, 2016); si la misma identificación personal toma estragos en la sociedad actual, entonces más será su efecto en la propia identificación nacional.

Tomando en cuenta aspecto generacionales, es claro observar en un evento social o educativo; la presencia de apatía por el respeto a las normas sociales o el respeto a los símbolos patrios, se hace evidente. Y no solo por la significancia a los emblemas nacionales hacia cierto grupo generacional; también estas actitudes se presentan en la preferencia de productos, de compañías, de actividades; lo que hace que la identidad por 
la nación transcienda a aspecto territoriales y culturales (Heat y Tilley, 2004). Señala Rodríguez (2020) la parte esencial de la identidad nacional como pilar, base y cimiento social, psicológico y personal; el sentimiento que genera ser parte de una nación, respetarla y emocionarse es resultado de la pertinencia social (Smith, 1997; Espinosa y Tapia, 2011). El valor adjudicado por la persona a la simbología, a la historia, a la cultura que lo hace pertenecer a un grupo y priorizar el ser colectivo, son elementos del autoconcepto colectivo (Rodríguez, 2020).

Y son estos mismos elementos los que pueden acercar o alejar al sujeto del grupo. De acuerdo a las características etarias de la población más joven, se encuentran sujetos preocupados más por la información y por sociedades digitales, que por lo que les rodea, estos son los actuales jóvenes entre 25 y 10 años de edad, que en su mayoría son la población estudiantil de secundaría y universidad, de acuerdo a Vicuña (2014) en Perú la identidad nacional de los jóvenes atiende a "indicadores socioculturales, símbolos patrios y étnicos" y que en estos se encuentran fuertes diferencias sobre el sentimiento de tolerancia.

Aún más, cuando en el país los aspectos culturales ancestrales son característicos por su riqueza artística, arquitectónica además de "la lengua, la religión, el modo de vida, forma de organización, costumbres, normas" (Rodríguez, 2020). El mismo autor señala, los acuerdos y estrategias ejecutadas por el Estado para transformar la marca del país, ya desde el 2002 los sectores económico y turístico, siendo el más reciente el fortalecimiento de la nación para los ciudadanos con la campaña "Casa Perú". De acuerdo a los esfuerzos del gobierno por fortalecer el sentido de pertenencia y la preferencia por las costumbres, cultura, actividades económicas, empresas, e inversiones del país, y desde un aspecto generacional, el presente artículo busca determinar la percepción de identidad nacional en jóvenes con características posmillennials, específicamente de pregrado de la Universidad Católica Santa María de la ciudad Arequipa, como resultados de la investigación llevada a cabo por Rodríguez (2020).

\section{Fundamentación teórica}

La identidad nacional como mecanismo para el desarrollo social de las personas, se fundamenta en aspectos psicológicos, educativos y sociales que hacen forman una imagen del sujeto a partir de los elementos o complementos únicos atributos del espacio o medio 
que le rodea (Espinosa, Freire y Ferrándiz, 2016). Para el caso del Perú, la identidad nacional en un concepto cambiante y que se encuentra en continua construcción (Portocarrero, 2015), aun cuando Yamamoto (2000) incluye esos elementos que permiten acercar la definición de identidad nacional, detalla la compleja relación resultante del sentimiento de pertinencia con un ámbito geo político, anexando la inclusión de todos esos elementos culturales, lengua, cultura política, valores, historia, recursos naturales, creaciones artísticas, diversidad étnica, los cuales hacen una amalgama compleja y sistemática de los significa un gentilicio o nacionalidad.

Detalla Rodríguez (2020) el confrontamiento de fenómenos sociales como el racismo interno en un país, el cual es muy marcado en el Perú "el rechazo de la sociedad continúa hacia lo indígena. Un claro ejemplo es la clase media limeña, que llega a considerarse a sí misma como lo que debe ser la sociedad". Desde este aspecto, el sentirse parte integrante y activo de una nación es valorar y tener respeto por la historia y lo que se quiere proyectar al futuro y al resto de países, lo cual ejerce influencia sobre el valor al patrimonio, a las normas, la cultura, las lenguas nativas, y las diferencias de los grupos humanos que conforman e integran la nación. (Rodríguez, 2020). El autor precitado realiza un esquema de los componentes de lo que es la identidad nacional haciendo referencia Guerrero (2017).

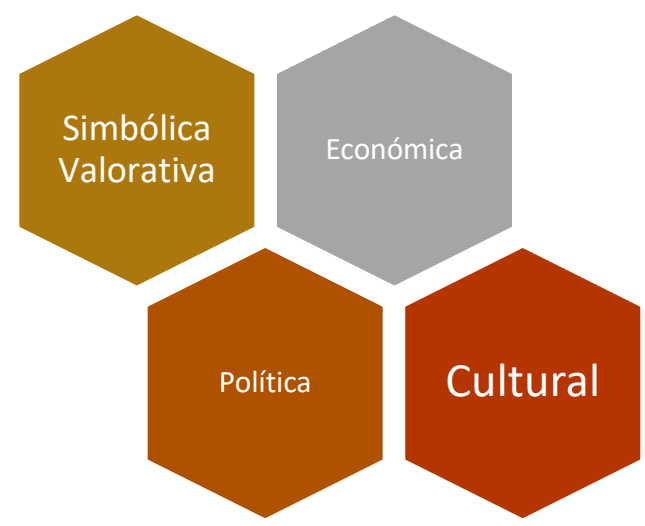

Fuente: Rodríguez (2020)

Simbólica valorativa: su carácter patriótico por ser "nacionalidad", conformado por los símbolos patrios, los deberes y derechos de la carta magna de la nación. (Rodríguez, 2020)

Económico: disposición y uso mecanismo económicos para el desarrollo del país, entre los cuales se contempla en uso de los recursos naturales y productos para entrar y 
mantenerse en el mercado económico, desde la competitividad, las relaciones y el libre comercio. (Rodríguez, 2020)

Política: visto de la autonomía de los integrantes del país por las decisiones en cuanto a su soberanía y el reconocimiento de ser una sociedad democrática y representativa en organizaciones mundiales y en los mecanismos para mantener el estado de derecho. (Rodríguez, 2020)

Cultura: protección y reconocimiento de los grupos y manifestaciones culturales únicas del país. Respeto a la diversidad y todas sus creaciones. (Rodríguez, 2020)

\section{Metodología}

Con una metodología de enfoque cuantitativo de diseño no experimental y transversal de nivel descriptivo, se empleó un cuestionario de treinta y seis preguntas cerradas con respuestas policotomicas de escala Likert: 1 "Totalmente en desacuerdo", 2 "En desacuerdo", 3 "Ni de acuerdo, ni en desacuerdo", 4 "De acuerdo" y 5 "Totalmente de acuerdo", el cual estuvo constituido por cuatro dimensiones: valorativa-simbólica, económica, política y cultural, expresadas en la tabla de operacionalización. La muestra estuvo conformada por 376 estudiantes con edades comprendidas entre los 14 y 25 años, los cuales fueron seleccionados de forma aleatoria con un nivel de confianza de $95 \%$ de una población de 16,432 estudiantes matriculados de la Universidad Católica Santa María de la ciudad de Arequipa. El instrumento fue validado por un juicio de expertos, por medio de la validez de contenido. Con el uso de programa SPSS se organizaron y presentaron los datos en tablas y gráficos porcentuales.

Tabla 1. Operacionalización de la variable

\begin{tabular}{|c|c|c|}
\hline \multirow{8}{*}{$\begin{array}{l}\text { Identidad } \\
\text { nacional }\end{array}$} & \multirow{3}{*}{$\begin{array}{l}\text { Simbólica } \\
\text { valorativa }\end{array}$} & Orgullo de la nacionalidad \\
\hline & & Identificación con símbolos nacionales \\
\hline & & Conocimiento y valoración de los deberes y derechos \\
\hline & \multirow{3}{*}{ Económica } & Autoridad sobre recursos nacionales. \\
\hline & & Dirección del desarrollo económico \\
\hline & & $\begin{array}{l}\text { Puesta en valor de productos nacionales en relación a } \\
\text { productos extranjeros }\end{array}$ \\
\hline & \multirow{2}{*}{ Política } & Posición frente a intervenciones externas \\
\hline & & Reconocimiento del país como demócrata \\
\hline
\end{tabular}


Álvaro Adrián Rodríguez Barriga

\begin{tabular}{|c|c|}
\hline & Posición frente a mecanismos para el cambio social \\
\hline \multirow{3}{*}{ Cultural } & Conciencia de la pluriculturalidad nacional \\
\hline & Valoración del patrimonio histórico nacional \\
\hline & $\begin{array}{l}\text { Posición en relación al efecto de la globalización en la cultura } \\
\text { nacional }\end{array}$ \\
\hline
\end{tabular}

Fuente: Rodríguez (2020)

\section{Resultados y Discusión}

Atendiendo a las características sociales de los estudiantes les fue solicitado indicar su lugar de nacimiento, aun cuando la institución tiene sede en Arequipa, esta recibe estudiantes de varias ciudades del país como: Moquegua (10,9\%), Tacna (9\%), Puno (6,4\%), Lima (5.9), Cuzco (4,8\%) y otras ciudades (7,7\%), el restante (55,3\%) son naturales de la ciudad Arequipa. Los resultados de la encuesta serán resumidos atendiendo a las percepciones más relevantes o dónde los estudiantes fueron positiva y negativamente decididos. Respecto al orgullo nacional sobre la historia del país más del $75 \%$ de los encuestados se sienten orgullosos por cada uno de los momentos históricos que marcaron la historia de su país, además de evidenciar con 70,4\% su manifestación abierta de la nacionalidad. (Figura 1)

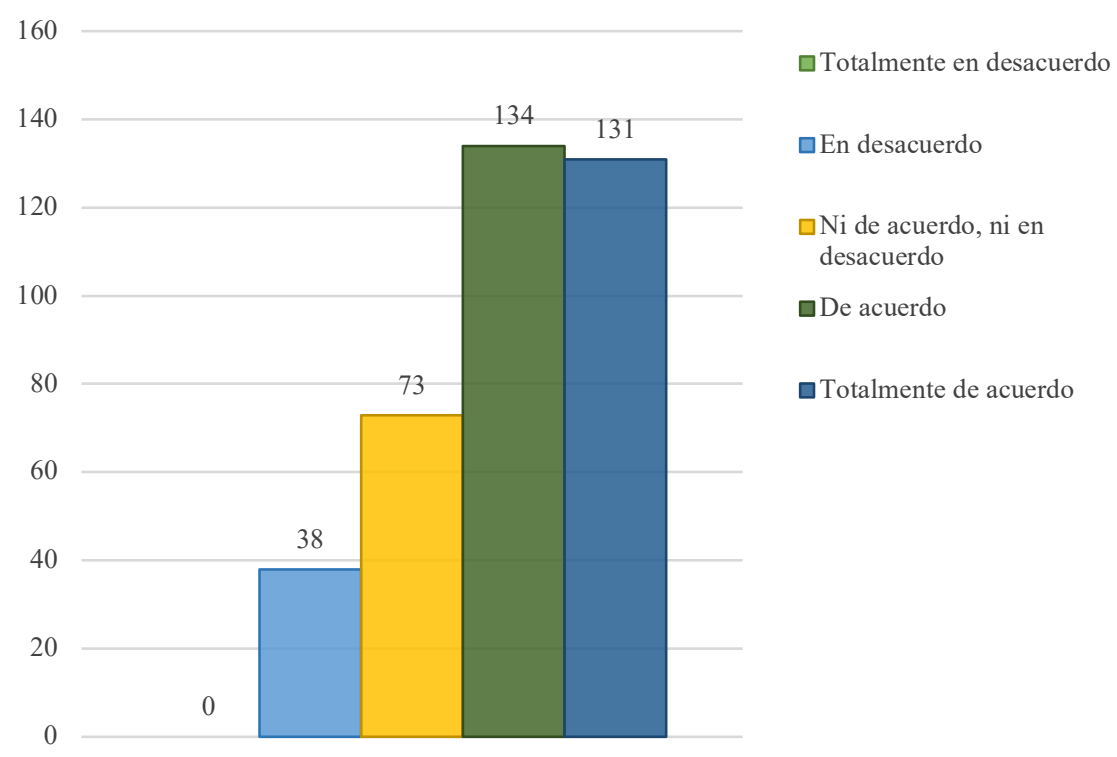

Figura 1. Manifestación abierta de la nacionalidad. Rodríguez (2020) 
Más, sin embargo, para la dimensión símbolos patrios, más de $80 \%$ de los estudiantes considera una pérdida de tiempo cantar el Himno Nacional. (Ver Figura 2). Pero a pesar de esta valoración negativa, indican con un $76,8 \%$ que el diseño, color y forma de los simbolos patrios son más hermosos que el resto de paises; y un 70,4\% consideran el uso de escarapela o prendas como señal de la peruanidad.

Siguiendo en la dimensión la mayoria de los estudiantes señalan la resposnabilidad compartida entre ciudadanos y gobernantes por el desarrollo historico del país, e igualmente señalan el papel fundamental de estos últimos en desarrollo del país; pero detallan con preocupación con un 78,2\% la desvalorización y irrespeto por la constitución del país, el cual consideran un documento incierto (debido a los recientes hechos de protestas por la vacancia presidencial con la consigna de reforma constitucional).

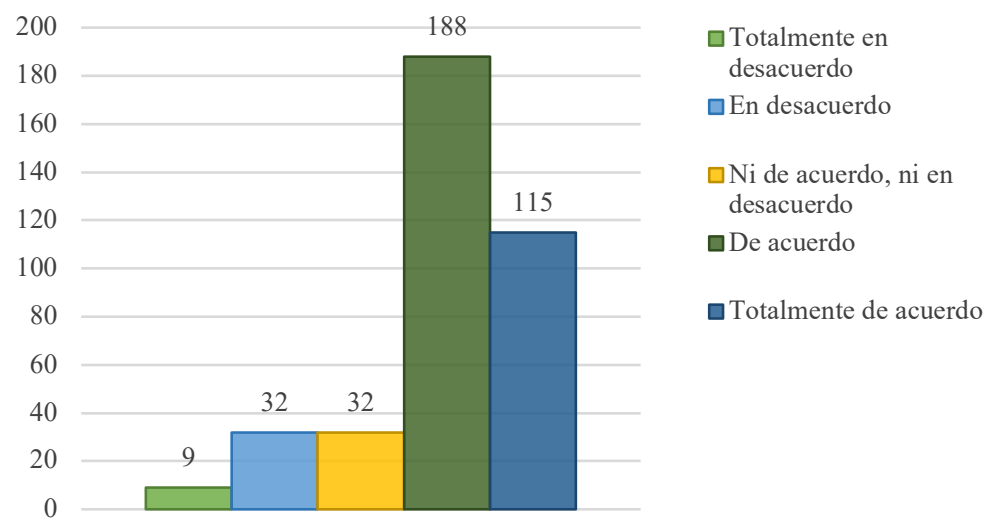

Figura 2. Considera una pérdida de tiempo cantar el Himno Nacional. Rodríguez (2020)

Para la dimensión economia más del 59\% de los estudiantes reconocen el potencial economico del pais, y más por los recursos naturales existentes en la nación, aun cuando se encuentran en una posición incierta sobre: las ventajas del posicionamiento geografico del pais y de la participación extranjera en beneficio del aparato economico. En cuanto al reconocimiento de superioridad de productos extranjeros sobre los nacionales $50,5 \%$ de los estudiantes prefiere trabajar para una empresa con sello internacional (Figura 3) 


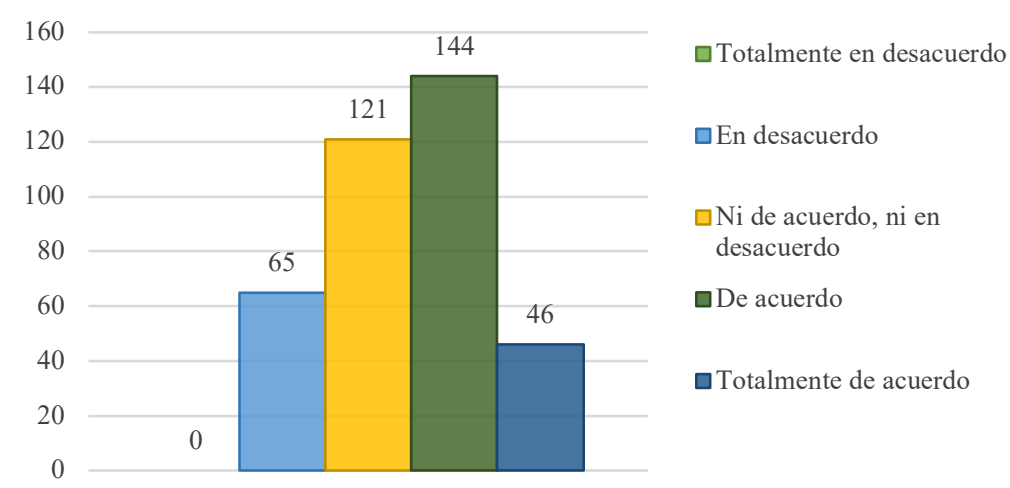

Figura 3. Considera más relevante trabajar en una empresa extranjera que en una peruana. Rodríguez (2020)

En cuanto a la dimensión política, para autonomía en decisiones políticas muestran una posición indiferente, pero consideran $(48,7 \%)$ que las decisiones en cuanto a la soberanía solo deben ser tomadas por los naturales del país, por conformar una nación democrática (49,5\%), por tanto, toman una posición indiferente en mecanismos como la revocatoria y/o el referéndum (Figura 4), a vez detallan que el uso de la violencia no es un mecanismo para el rechazo de decisiones gubernamentales $(51,1 \%)$, más sin embargo son neutral sus postura hacia un gobierno que usa medidas coercitivas cuando las estrategias se agotaron a la hora de resolver un conflicto.

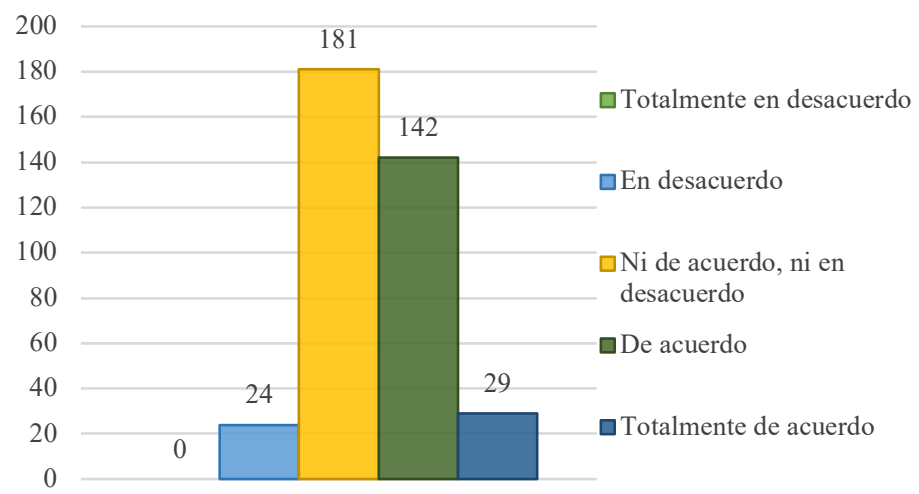

Figura 4. Instrumentos como la revocatoria y el referéndum ayudan a reforzar la democracia. Rodríguez (2020)

En cuanto a la dimensión cultura, se repite su postura neutral en asuntos de índole racial, ya que les es indiferente el reconocimiento de la diversidad de razas como integrante de la identidad nacional $(52,1 \%)$, además de señalar que es más valioso saber de arte extranjero que del nacional (53.5\%), y la desvalorización de la arqueología 
Álvaro Adrián Rodríguez Barriga

prehispánica al considerarla un impedimento para el desarrollo urbano (54.5\%), la apatía se hace presente al considerar indiferente aprender sobre las lenguas nativas $(46,8 \%)$; más contradicen esta respuesta al indicar con la aseveración que la diversidad cultural es ventaja competitiva frente a otros países $(51,3 \%)$ y sobre la percepción de responsabilidad sobre el sub desarrollo del país por Norteamérica y el Continente Europeo (50,6\%) (Figura 5)

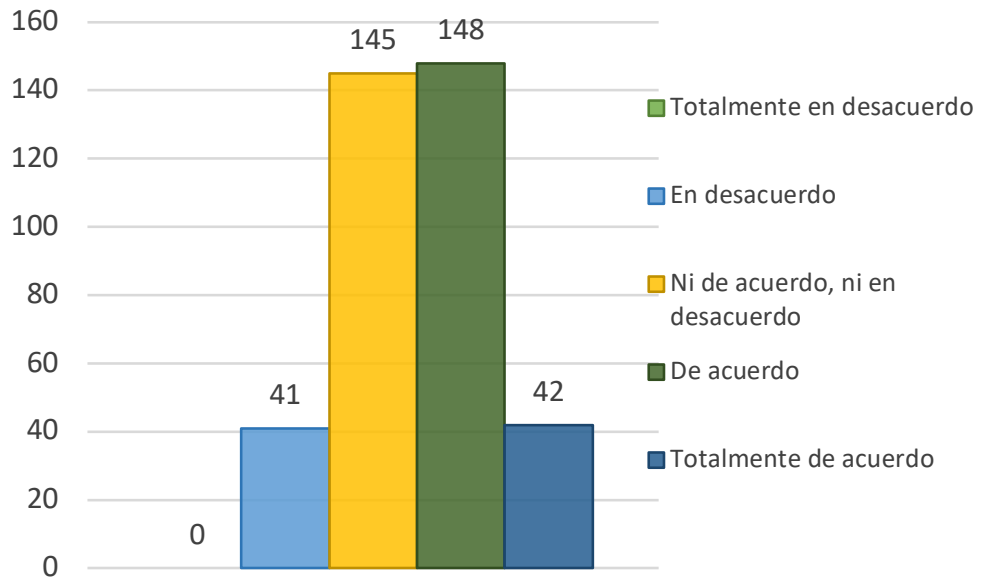

Figura 5. Considera adecuada una posición antinorteamericana y antieuropea por considerarlos responsables del subdesarrollo del país. Rodríguez (2020)

A raíz de estos resultados es evidente, la eficacia del modelo de Guerreo (2017) al concebir la identidad nacional en cuatro dimensiones, las cuales presenta perfectivas acertadas a los componentes que describen la identidad nacional, y en este caso la peruanidad, aun cuando fue necesario adaptar la operacionalización de las variables a la realidad del país en: políticas pública, derechos humanos, propiedad de la tierra, académicos y de interés propios. Por tanto, se asume la posición de por tratarse de una condición propia de un país, y sin incurrir en aspectos más específicos como la identidad local o regional.

La conformación de un estudio cuantitativo, para determinar la percepción de identidad nacional atiende a elementos cambiantes hasta dentro de un mismo país, o de países vecinos, y estas emergen de: la cultura, el arte, las costumbres, tradiciones, lenguaje, y de condiciones geográficas que son irrepetibles. (Verde, 2020; Rodríguez, 2020). Estando ante una generación, apática ante elementos importante de lo que significa ser peruano, desvaloriza la importancia de elementos históricos solo por no ser cónsonos con la realidad del mundo en cuanto al desarrollo urbanístico y a la relevancia de conocer todos los aspectos de la cultura, por no ser los tópicos importantes para el 
Álvaro Adrián Rodríguez Barriga

desenvolvimiento en esta sociedad de la información; las inconsistencias las presentan entre dimensiones y más de los indicadores donde la indiferencia se hace presente: autonomía política, medidas coercitivas del gobierno, mecanismos democráticos, aprendizaje de las lenguas nativas y la diversidad étnica.

\section{Conclusión}

Pero es notable, que, en términos generacionales, el grupo estudiado, es más indiferente y neutral a condiciones de suma importancia para identificarse como ser peruano, además de las inconsistencias entre cada respuesta, solo puede ser evidencia del desconocimiento hacia el propio país, o la poca valorización de la identidad cultural, a los recursos naturales beneficio de la ubicación geográfica, respeto a los símbolos patrios (como el himno nacional), la diversidad cultural.

El joven peruano que en su mayoría conforman la población estudiantil a nivel universitario da más importancia a conocimientos culturales generales, que le permita entrar en el campo competido muestra de ellos su preferencia por trabajar en empresas extranjeras, conocer de arte y usar productos de reconocida superioridad extranjera. Los posmillennials de la Universidad Católica Santa María de la ciudad de Arequipa son estudiantes indiferentes a la valoración nacional, su interés se centra en la globalización cultural, en la practicidad de los recursos para consolidar una posición competitiva a nivel económico; pero mantienen un sentimiento de orgullo al decir que son peruanos.

\section{Referencias Bibliográficas}

Cueto R., Espinosa A., Robles R. (2017) Narrativas sobre la sociedad peruana y la identidad nacional en universitarios peruanos. Revista Límite. Vol. 12, núm. 38. Universidad de Tarapacá Arica, Chile. ISSN: 0718-1361. Tomado de https://www.redalyc.org/pdf/836/83651038002.pdf

Espinosa, A. y Tapia, G. (2011). Identidad nacional como fuente de bienestar subjetivo y social. Boletín de Psicología, 102, 71-88.

Espinosa, A., Freire, S., y Ferrándiz, J. (2016). Identificación colectiva y bienestar en una comunidad rural de la costa norte del Perú. Revista De Psicología, 34(1), 201-219. https://doi.org/10.18800/psico.201601.008 
Álvaro Adrián Rodríguez Barriga

Guerrero Huamán, J. (2017). Desarrollo del área de historia del Perú y la formación de la identidad nacional en los alumnos del II ciclo del ISTP Carlos Cueto Fernandini. (Tesis de doctorado) Universidad Nacional de Educación.

Heath A. y Tilley J. (2004). British National Identity and Attitudes towards Immigration. International Journal of Multicultural Societies. 7.

Pastor, G. (2016). Estudio sobre la afirmación de la identidad nacional en el Perú. Centro de Altos Estudios Nacionales. Vol. 2: 109-134. Tomado de: http://www2.congreso.gob.pe/sicr/cendocbib/con5 uibd.nsf/724B14F44EA54588 052582BE00770AD7/\%24FILE/ART4-G-Pastor.pdf

Portocarrero G. (2015) La urgencia por decir "nosotros". Los intelectuales y la idea de nación en el Perú republicano. Investigaciones Sociales. Online ISSN: 1818-4758

Rodríguez, A. (2020). Notoriedad de la marca Perú y su relación con la formación de identidad nacional en los estudiantes de pre grado de la Universidad Católica Santa María, Arequipa, 2020. (Tesis de Doctorado) Universidad Católica Santa María. Perú.

Smith, F. (1997). Historia de la Antropología, vol. I: Historia de la Antropología. Indigenista, México y Perú. PUCP Fondo editorial, Lima, Perú.

Verde Rivera, V. (2020). Estudio sobre la identidad nacional y su influencia en la autorrealización de los estudiantes de educación primaria y problemas de aprendizaje de la facultad de educación de la Universidad Nacional José Faustino Sánchez Carrión, Lima, 2020. (Tesis de Maestría). Universidad Nacional José Faustino Sánchez Carrión.

Vicuña L. (2014). Sentimientos de identidad nacional de púberes y adolescentes de la provincia de Tarma y de la ciudad de Lima. Revista de Investigación en Psicología. https://www.researchgate.net/publication/265524857 Sentimientos de identidad _nacional_de puberes_y adolescentes de la provincia_de Tarma y de la_ciud ad de Lima

Yamamoto, J. (2000). Información e identidad: autoestima nacional, identidades sociales e identidad nacional. Separatas del curso de Psicología Social. Lima: Facultad de Letras y Ciencias Humanas, PUCP. 